\title{
Measurement of Partial Discharge Activities within Two Artificial Spherical Voids in an Epoxy Resin
}

\author{
H.A. Illias \\ Department of Electrical Engineering \\ University of Malaya \\ 50603 Kuala Lumpur \\ Malaysia
}

\author{
G. Chen and P.L. Lewin \\ The Tony Davies High Voltage Laboratory \\ University of Southampton \\ Southampton \\ SO17 1BJ United Kingdom
}

\begin{abstract}
Measurement of partial discharge activities within insulation systems has been extensively performed. It has been widely used in the performance assessment of an insulation system and for insulation diagnostics. Since a void cavity is one of the potential sources of $P D$ activity in insulation which may cause degradation and breakdown, it is very important to study PD events associated with void cavities solid dielectric insulation. In this work, the measurement of PD activity has been performed on test samples consisting of two spherical voids within an epoxy resin. Two voids of same and different sizes are positioned close to each other and in parallel with the direction of the applied field within the epoxy. Through comparison of the experiment data for PD events within a single void in the epoxy, single and multiple discharge sources from different voids can be clearly distinguished.
\end{abstract}

\section{INTRODUCTION}

It is very important to study PD events within void cavities that exist in solid dielectric insulation systems because a void cavity is one of the potential sources of PD activity in insulation material and ultimately may cause degradation and breakdown of the insulation.

The measurement of PD events within two cylindrical voids which are positioned in parallel and series with the direction of the applied field in a dielectric material has been reported in other previous work [1]. The measurement results show that the PD inception field was found to be higher when two voids are arranged in series with each other and with the applied field direction compared to when they are positioned in parallel to each other. This is due to a lower maximum electric field magnitude for the serially-arranged voids, resulting in a higher applied voltage required to reach the inception field [13]. Because of the reduced effective material thickness along the regions containing serially-arranged voids with the direction of the applied field, the breakdown strength of the material obtained is also lower.

In this work, the measurement of PD activity has been performed on test samples consisting of two spherical voids in an epoxy resin, which is widely used as the dielectric material in power apparatus. Two voids of the same and different sizes are positioned close to each other and in parallel with the direction of the applied field within the epoxy. Two parallel, cylindrical electrodes were used to ensure that a uniform electric field is applied across the samples. Through comparison with the measurement of PD events within a single void in the epoxy, single and multiple discharge sources from different voids can be clearly distinguished [4-11].

\section{Measurement OF PD ACtivities}

A typical measurement setup that has been used for PD experiment in the laboratory is shown in Fig. 1. The experiment consists of a high voltage supply, $V$, a coupling capacitor $C_{k}$, a test sample, a measuring impedance, a digital signal oscilloscope (DSO), a PD detector and a USB controller which is connected to a personal computer (PC) $[10,12,13]$. A charge is transferred from $C_{k}$ to the electrode on the test object when a PD occurs. The measuring impedance and the PD detector detect PD signals and the data is sent through fibre optic cables to the PC. The DSO is used to capture PD signals from the measuring impedance as a function of time.

The test sample is made of an epoxy resin which is embedded with two artificial spherical voids of diameters, $d_{l}$ and $d_{2}$, located side by side to each other with a gap separation, $g$ in the middle of the material (Fig. 2). Initially, air bubbles were injected into two small epoxy blocks before curing to obtain a spherical void in each and the blocks were then cast into a larger epoxy block. Curing for 24 hours at ambient temperature, post curing for 4 hours at $90^{\circ} \mathrm{C}$ and cooling to ambient temperature for 2 hours was then undertaken prior to any experimentation. The top surface of the sample was connected to a high voltage electrode while the bottom surface is connected to a ground electrode. The whole test sample and the electrodes were immersed in mineral oil. A $50 \mathrm{~Hz}$ ac sinusoidal voltage (voltage amplitude, $U_{a p p}$ ) was applied and PD data over 1500 applied voltage cycles was recorded.

Table 1 shows the dimensions of the test samples that have been created. Due to the difficulty in creating artificial spherical voids and controlling the positions of the smaller

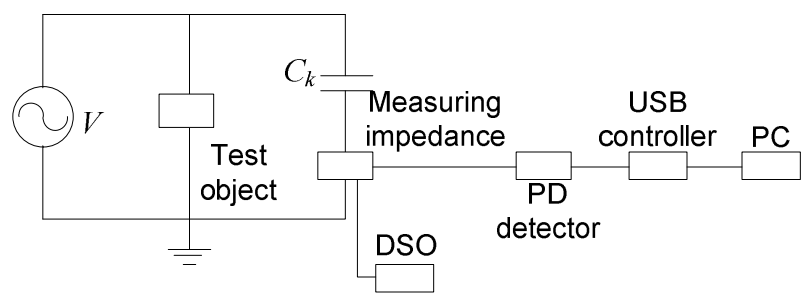

Fig. 1. PD experiment setup. 


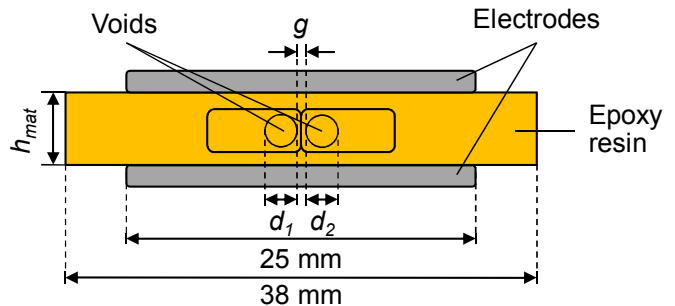

Fig. 2. The test sample and the electrodes.

TABLE I

Samples Dimensions that have been Prepared for Fig. 2

\begin{tabular}{|l|c|c|c|c|c|}
\hline Sample & $d_{1}(\mathrm{~mm})$ & $d_{2}(\mathrm{~mm})$ & $g(\mathrm{~mm})$ & $h_{\text {mat }}(\mathrm{mm})$ & $U_{\text {app }}(\mathrm{kV})$ \\
\hline 1 & 2.7 & 2.7 & 0.6 & 4.0 & 22 \\
\hline 2 & 3.0 & 3.6 & 0.7 & 5.4 & 20 \\
\hline
\end{tabular}

epoxy block, all samples have different dimensions. However, comparison of PD events between different samples can still highlight common features that occur due to the close proximity of the two voids.

\section{PD MEASUREMENT RESULTS}

\section{A. PRPD patterns}

Fig. 3 shows PRPD patterns for the PD activities of test samples 1 and 2, which consist of two voids positioned close to each other and located horizontally with reference to the direction of the applied field. From these figures, it can be seen that discharges from different voids can be distinguished clearly, as marked with red dashed lines. When PDs occur within two same sized voids, as shown in Fig. 3a, PDs can be seen to occur at a high repetition rate within one of the voids, by referring to PD pattern that occurs near the minimum charge magnitude (void 1). However, only a few PDs occur within the other void, which can be seen from PD pattern that resembles a 'tortoise-like' curve (void 2). In sample 1, discharge events are dominated by only one void.

From the simulation model that has been developed by the same authors, when a PD occurs within one of the voids in sample 1 until its field becomes less than the extinction field, then, the field in the other void decreases. Hence, there is a reduced number of PDs per cycle obtained from the second void. The cross section plot of field along the $\mathrm{x}$-axis through this void becomes asymmetrical after a PD occurs in the first void, where the field is lowest at the surface region nearest to the void surface. This will affect charge distribution along the second void surface, where it is reduced on the surface perpendicular to the applied field. These charges, which may act as the initial free electrons for the next PD to occur, are reduced in terms of surface emission. Thus, the electron generation rate becomes lower, increasing the time delay of a PD occurring once the inception field in this void is exceeded. This results in a lower number of PDs per cycle and a 'turtle like' PD pattern is generated by the void.

Referring to Fig. 3b, when PD events occur within two voids of different diameters that are positioned near and in parallel to each other with the direction of the applied field, two clearly separated 'tortoise-like' patterns can be seen. A region of higher minimum and maximum PD charge magnitudes, as marked with dashed red lines in Fig. $3 \mathrm{~b}$ is believed to be due to the larger void diameter (void 2). A larger void diameter allows the head of the electron avalanche to grow bigger and longer in the direction of the applied field [13]. The number of PDs per cycle occurring from voids 1 and 2 are similar, unlike the PD pattern shown in Fig. 3a.

From the simulation, when the size of void 2 is larger than void 1 , the field in void 2 is lower than void 1 . When a PD occurs in void 1 (a smaller void), the field in void 2 is not significantly affected by the field reduction of void 1 . Thus, the likelihood of a PD occurring within void 2 is not significantly altered since the charge distribution along the void 2 surface does not significantly change from that before the PD occurs in the other void.

If a PD occurs in void 2 (the larger void), the field in void 1 is greatly reduced due to the reduced field in void 2 . However, the likelihood of a PD occurring in void 1 is still high if the inception field is exceeded. The field distribution in void 1

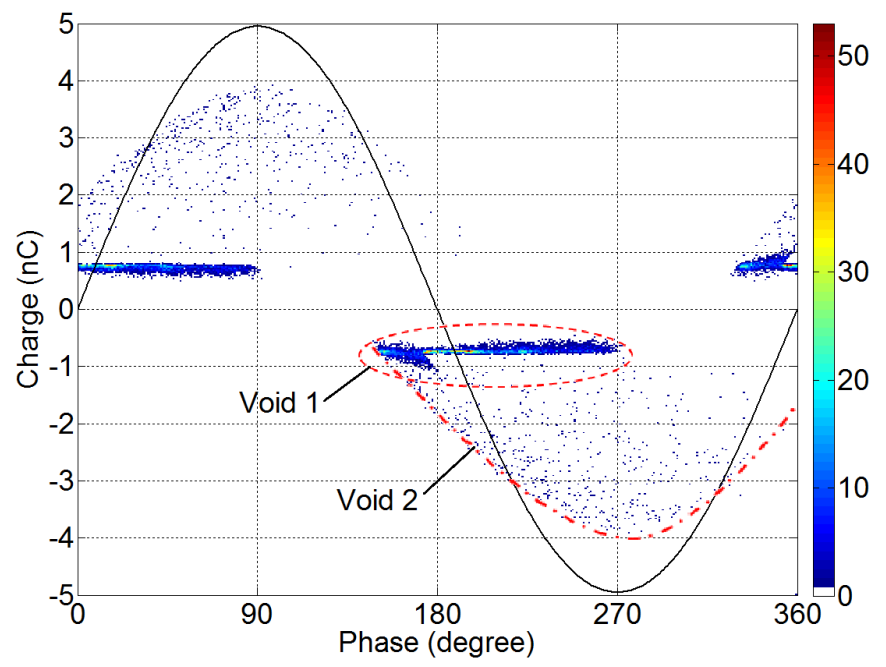

(a)

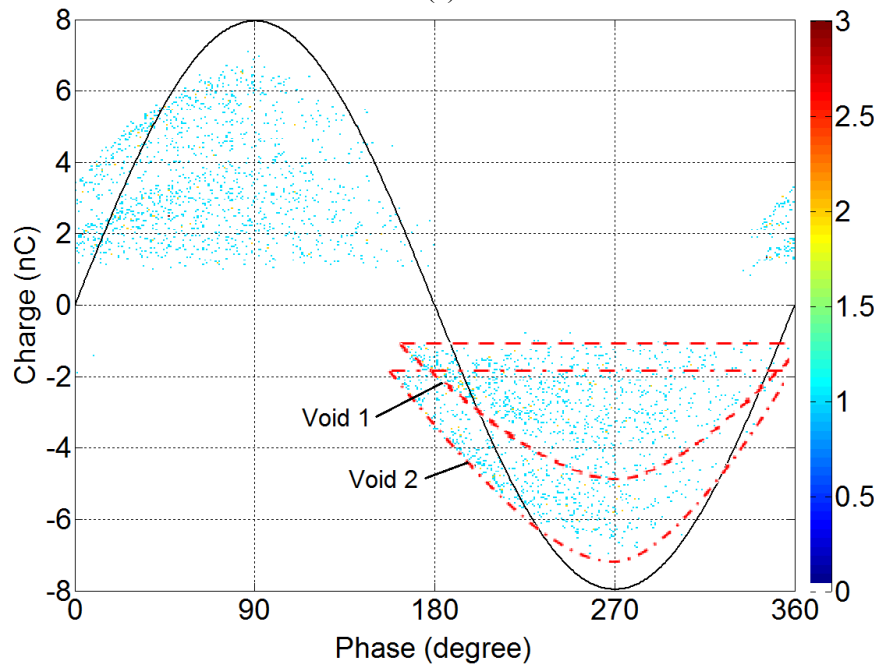

(b)

Fig. 3. PRPD patterns for PD activities within two voids positioned close to each other and horizontally with the direction of the applied field: (a) two same void sizes (sample 1), (b) two different void sizes (sample 2) (Red dashed lines show example of PD pattern segregation from different voids). 
becomes much higher in its centre and consequently the charge density is higher at the top and bottom of void 1 surface. Hence, initial free electrons will be available to initiate a PD in void 1 should the inception field be exceeded.

The PRPD pattern for PD events within a single void is shown in Fig. 4. Unlike Fig. 3, only a pair of 'turtle-like' patterns can be seen in Fig. 4, which indicates that PDs are occurring only within one void. Thus, PD activities within single and multiple voids can be clearly distinguished through analysis of PRPD patterns.

\section{B. Pulse sequence analysis}

The sequence of individual PD occurrences one after another can be observed using pulse sequence analysis (PSA) plots $[11,14]$. PSA records the time and applied voltage amplitude of each PD occurrence. Using these data, scatter plots of the time interval between consecutive discharges and the difference in the applied voltage amplitude between two PD occurrences can be obtained. These plots provide another way of analyzing PD data besides PRPD patterns.

Figs. 5 and 6 show the scatter plots of time and applied voltage differences between consecutive discharges obtained from PD events within two voids of same and different sizes and PD activity for a single void. In these figures, $\Delta U$ and $\Delta t$ are the voltage and time differences between two consecutive PD occurrences and $n$ is the $n$-th PD event. From Fig. 5, the regions of $\Delta U_{n}$ vs. $\Delta U_{n-1}$ and $\Delta t_{n}$ vs. $\Delta t_{n-1}$ that are close to zero indicate PDs are occurring within two voids, compared to PDs occurring within a single void (Fig. 6). However, there are no simultaneous PDs occurring within two voids because the minimum time differences between consecutive discharges are 8.2 us and 10.2 us from Fig. 5a and $5 \mathrm{~b}$ respectively. The minimum $\Delta t$ for PD events within a single void is $2.2 \mathrm{~ms}$.

When comparing Fig. $5 \mathrm{~b}$ with Fig. 6 , both of them have similar scatter plot patterns of $\Delta U$, by referring to the obvious 'triangle' and 'square-like' patterns. The difference between these two figures is the 'empty space' near $\Delta U$ of $0 \mathrm{kV}$ in Fig. $5 \mathrm{~b}$ compared to Fig. 6 . Since there is only one PD event within

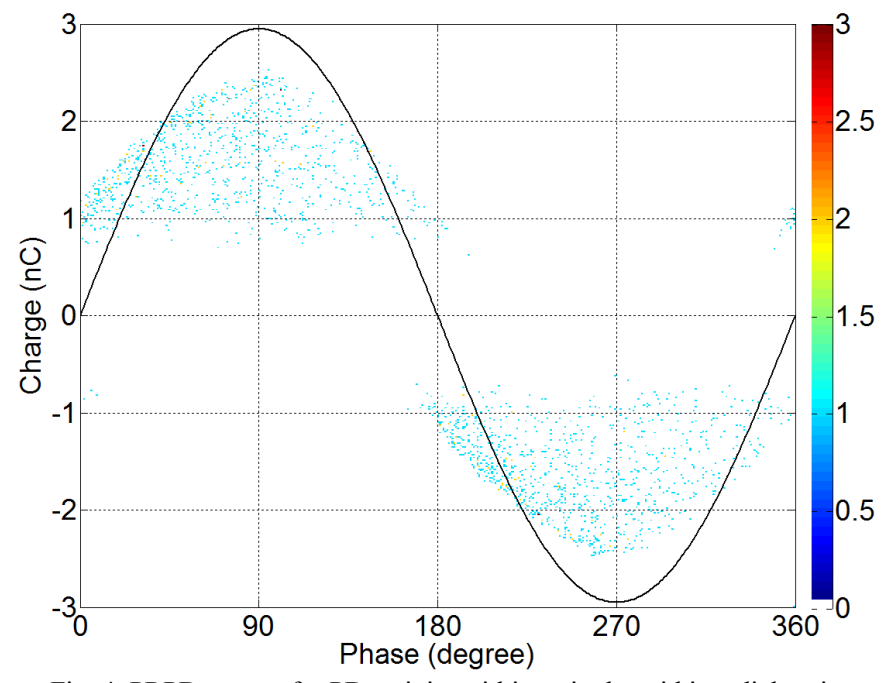

Fig. 4. PRPD pattern for PD activity within a single void in a dielectric material. a single void which affects the whole void space every time a PD occurs, the field in the void requires a certain time to recover its field until it exceeds the inception field again for the next PD to occur. Thus, the 'empty space' around $\Delta U$ of 0 $\mathrm{kV}$ and $\Delta t$ of $0 \mathrm{~ms}$ is visible in Fig. 6. However, in a two-void sample, after a PD has occurred within one void, a PD can occur within another void provided that the inception field is exceeded. This is why there is no obvious 'empty space' near $\Delta U$ of $0 \mathrm{kV}$ and $\Delta t$ of $0 \mathrm{~ms}$ in Fig. 5b. Fig. 5a is a bit different because PDs occur rapidly within one void but less frequently within the other void. Therefore, from PSA plots, PD activities within single and multiple voids can also be clearly distinguished.

\section{Cycle to cycle behaviour of PD events}

The occurrence of PD events for 10 applied ac voltage cycles obtained using a digital signal oscilloscope (DSO) is shown in Fig. 7, where each 'spike' of voltage amplitude against time, is a PD occurrence. In Fig. 7a, for PD events that
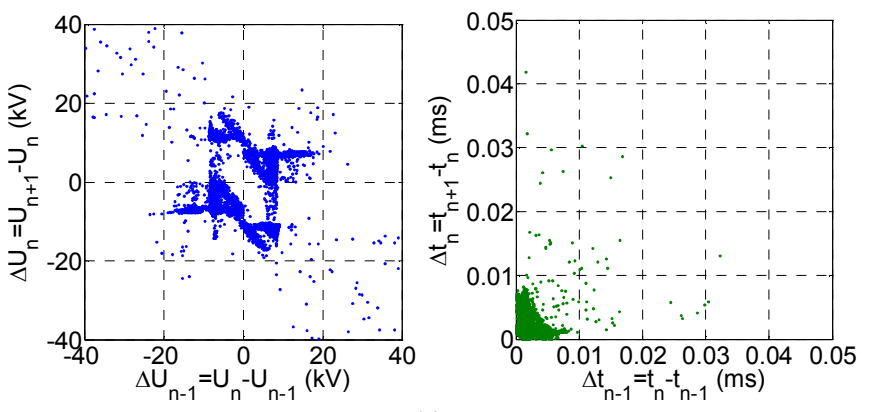

(a)
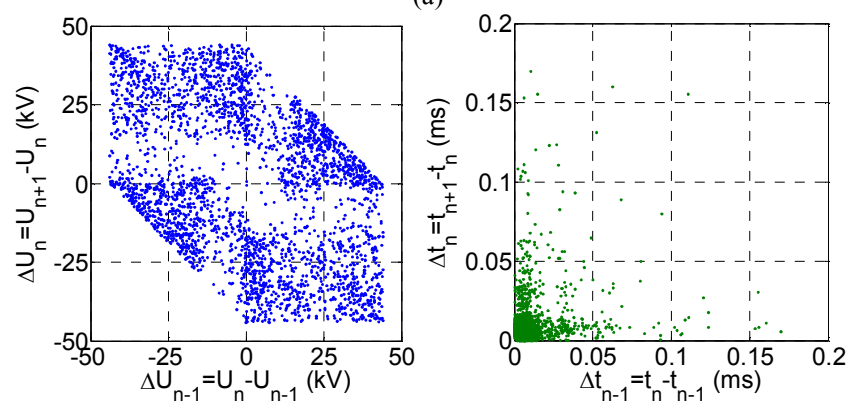

(b)

Fig. 5. Pulse sequential analysis (PSA) plots for PD events within two voids located close to each other and horizontally with the applied field direction in an epoxy resin: (a) two different void sizes (20 kV applied voltage), (b) two same void sizes ( $22 \mathrm{kV}$ applied voltage).
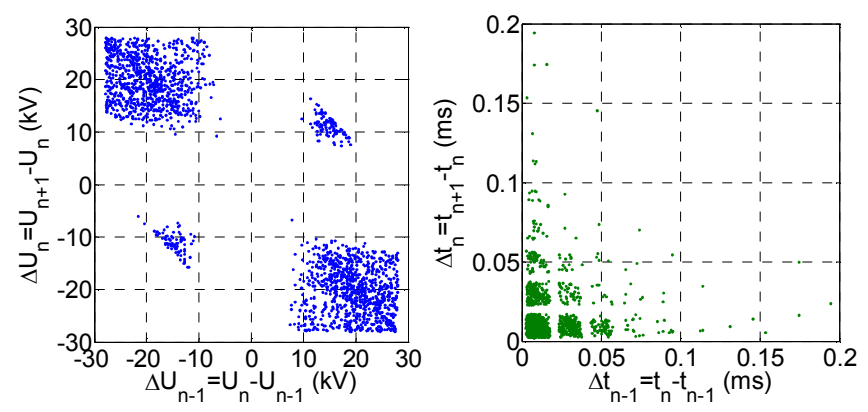

Fig. 6. Pulse sequential analysis (PSA) for PD within a single void in an epoxy resin (14 kV applied voltage). 
occur within two same-sized voids (sample 1 in Table 1), PDs occur quite rapidly with lower amplitude over each applied voltage cycle. While in Fig. 7b, for PD events that occur within two different-sized voids (sample 2 in Table 1), PDs can be seen to occur less frequently but with higher amplitude at each applied voltage cycle.

Unlike PRPD patterns and PSA scatter plots, it is quite difficult to differentiate whether PDs occur within single or multiple voids in a dielectric material without analysis of cycle to cycle behavior of PD events. However, it is possible to see whether any simultaneous discharges occur or not within the two void samples from PD signals obtained using the DSO. From the signals obtained through the experiment, no simultaneous discharges occur from the two voids. As expected, it is quite impossible for PDs to occur at or almost the same time from multiple voids.

If a sample containing two voids located far from each other within a dielectric material, it is expected that PD patterns from both voids will overlap each other completely. This is due to the independent electric field distribution of the voids so that when a PD occurs within either one of the voids it has no effect on the other one.

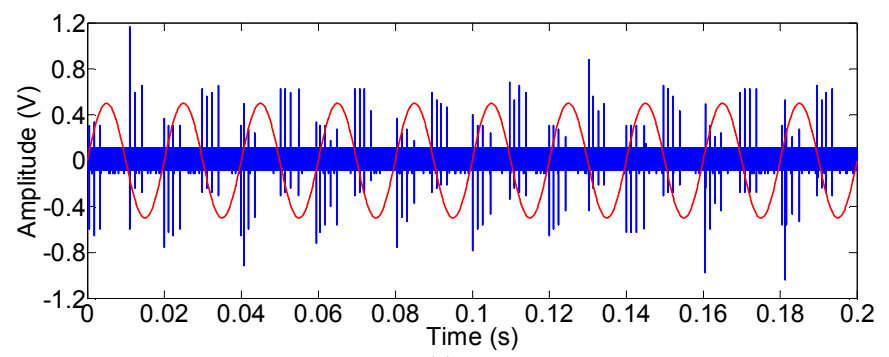

(a)

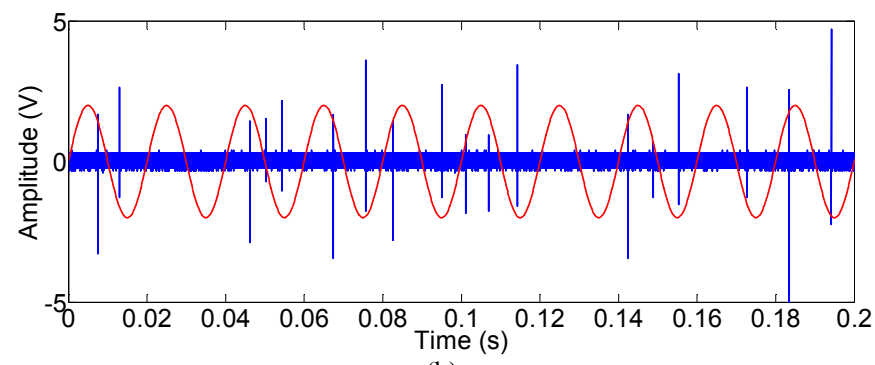

(b)

Fig. 7. Signals obtained using a digital signal oscilloscope (DSO) from samples consisting of two voids with (a) same size and (b) different size.

\section{CONCLUSIONS}

The measurement of PD activities within two voids that are located very close to each other and in parallel with the direction of the applied field in a dielectric material has been undertaken. From the measurement results obtained, PDs occurring within two different voids can be clearly distinguished. PD events occurring within two same and different size of voids can be differentiated from PDs occurring within a single void in a dielectric material through phase resolved partial discharge (PRPD) patterns and pulse sequential analysis (PSA) plots. There is no evidence of discharges occurring simultaneously from the two different voids based on PD signals that have been captured using a digital signal oscilloscope. Moreover, the shortest time interval between consecutive discharges from different voids is of the order of 10 microseconds. Further work will consider measurement of PD events within two voids positioned on top of each other in the direction of the applied field and also further comparison of measurement and simulation results for PD events within two voids.

\section{REFERENCES}

[1] J. Seung-Ik, S. Doe-Sung, Y. Do-Hong, H. Key-Man, and H. Min-Koo, "A study on the partial discharge characteristics according to the distribution pattern of voids within an insulation," International Conference on Conduction and Breakdown in Solid Dielectrics, pp. 398$402,1995$.

[2] A. A. Hossam-Eldin, S. S. Dessouky, S. M. El-Mekkawy, and R. A. A. El-Aal, "Analysis and simulation of field distribution in micro cavities in solid insulating materials," Conference on Electrical Insulation and Dielectric Phenomena, pp. 792-796, 2007.

[3] M. E. Ghourab and S. M. El-Makkawy, "Analysis of electric field distribution in cavities within solid dielectric materials," Conference on Electrical Insulation and Dielectric Phenomena, pp. 155-160, 1994.

[4] L. Niemeyer, "A generalized approach to partial discharge modeling," IEEE Transactions on Dielectrics and Electrical Insulation, vol. 2, pp. 510-528, 1995.

[5] F. Gutfleisch and L. Niemeyer, "Measurement and simulation of PD in epoxy voids," IEEE Transactions on Dielectrics and Electrical Insulation, vol. 2, pp. 729-743, 1995.

[6] R. Schifani, R. Candela, and P. Romano, "On PD mechanisms at high temperature in voids included in an epoxy resin," IEEE Transactions on Dielectrics and Electrical Insulation, vol. 8, pp. 589-597, 2001.

[7] A. Cavallini, R. Ciani, M. Conti, P. F. H. Morshuis, and G. C. Montanari, "Modeling memory phenomena for partial discharge processes in insulation cavities," IEEE Conference on Electrical Insulation and Dielectric Phenomena, pp. 723-727, 2003.

[8] C. Forssen and H. Edin, "Partial discharges in a cavity at variable applied frequency part 2: Measurements and modeling," IEEE Transactions on Dielectrics and Electrical Insulation, vol. 15, pp. 1610-1616, 2008.

[9] P. Morshuis, A. Cavallini, G. C. Montanari, F. Puletti, and A. Contin, "The behavior of physical and stochastic parameters from partial discharges in spherical voids," International Conference on Properties and Applications of Dielectric Materials, vol. 1, pp. 304-309, 2000.

[10] H. Illias, G. Chen, and P. L. Lewin, "Partial Discharge Behavior within a Spherical Cavity in a Solid Dielectric Material as a Function of Frequency and Amplitude of the Applied Voltage," IEEE Transactions on Dielectrics and Electrical Insulation, vol. 18, pp. 432-443, 2011.

[11] P. Rainer and B. Farhad, "Pulse Sequence Analysis - a diagnostic tool based on the physics behind partial discharges," Journal of Physics D: Applied Physics, vol. 35, pp. 25-32, 2002.

[12] H. Illias, G. Chen, and P. L. Lewin, "The influence of spherical cavity surface charge distribution on the sequence of partial discharge events," Journal of Physics D: Applied Physics, vol. 44, pp. 1-15, 2011.

[13] H. Illias, G. Chen, and P. L. Lewin, "Modeling of partial discharge activity in spherical cavities within a dielectric material," IEEE Electrical Insulation Magazine, vol. 27, pp. 38-45, 2011.

[14] D. Benzerouk, J. Menzel, and R. Patsch, "The role of accumulated charges in partial discharge processes," IEEE International Conference on Solid Dielectrics, pp. 552-555, 2007. 